\title{
Modelling the demand for learning resources in academic libraries
}

Jon Warwick

\begin{abstract}
System dynamics is a relatively young discipline having been in existence for just 50 years. With close links to systems theory it offers an holistic approach to modelling and focuses on the analysis of the feedback structures existing in complex systems that give rise to observed behaviour. This paper gives a description of some of the classic archetypal system structures that commonly occur in system dynamics models and interprets them within the context of modelling an academic library system. The paper summarises the process of modelling with system dynamics and gives examples of some behavioural insights that emerge from a simple interconnection of archetypes to form a qualitative model of part of a library system relating to loan and duplication policy.
\end{abstract}

\section{Introduction}

In 2007 the community of researchers and practitioners working within the field of system dynamics celebrated 50 years since the founding of the discipline (Sterman, 2007). Given its relatively short history, the study of system dynamics has made significant contributions to the understanding of system behaviour and policy formulation within a wide range of contextual areas including business, management, engineering, and medicine. That it has made an impact in so short a time is testimony to the value of the insights system dynamics can provide on the behaviour of complex systems, yet despite some interest in applying general systems theory to a number of education related problems there have been few applications of system dynamics within library and information science. In this paper, we illustrate some of the commonly recurring system dynamics feedback structures that have been observed over the last 50 years and show how these also occur in the description of academic library systems. Further, the paper illustrates the use that can be made of these system archetypes (as they are termed) in building qualitative models that help managers to appreciate the cause and effect relations that are at play in any complex system.

\footnotetext{
Author

Dr. Jon Warwick is a Professor of Educational Development in the Faculty of Business, Computing and Information Management at London South Bank University.

E-mail: warwick@1sbu.ac.uk

Received July 2008
}

Accepted August 2008 


\section{Taking a systems view}

The application of quantitative and qualitative modelling to the analysis of academic library systems has long been of interest to those responsible for setting and delivering library policy and those within the management science community who find the types of problems associated with modelling such systems challenging and fraught with difficulty.

The application of general systems theory to planning and resource allocation in higher education institutions has attracted a good deal of interest over the last 10 years or so. Suggested areas of application have included the development of training and education programmes, effecting organisational change within institutions and also the sector as a whole, understanding the nature of learning at the individual or organisational level and the development of course or learning support processes. The last of these is of particular interest here as course and learning support processes will include library and learning resources.

If, for the purposes of this paper, we restrict our discussion of library systems to consideration of loan and duplication policy within a university library then a starting point for analysis of the system (assuming that we are, in some way, trying to 'optimise' the loan and duplication policy) might be to recognise that it is a human activity system. In other words, it is the activity of the staff and students (actors and users) within the structure of the library system that give it meaning and that give rise to the behaviour that we observe and the statistical information we collect that describes that behaviour. If we continue down this line of argument then to understand the system requires that we understand the behaviour of the actors and users and this is where problems for the modeller begin to become apparent.

Human behaviour can sometimes be difficult to rationalise and even if we assume that behaviour is logical, how can we access the individual's perception of the system that will condition their response and generate action? We are all familiar with these sorts of interactions throughout everyday life: the demand for goods in a shop might be significantly reduced by initial excessive demand (and hence continual shortage of stock) as customers seek other suppliers or substitute goods, the arrival rate and behaviour of customers in a queue will be altered by the perceived waiting time or queue length, a newly opened stretch of road becomes choked with motorists trying to use it or, strangely, remains empty as motorists assume that it will be busy!

A further problem relates to describing the criteria that users adopt in deciding their best course of action - that is to say what is our definition of 'optimal' in setting policy for users? There are many metrics that might be used to give some indication of system performance and these would include, inter alia, the cost of providing the system, user satisfaction, response time to requests, book usage (circulation rates, number of reservations etc.). Unfortunately, this is really only part of the picture. User satisfaction could be seen as the crucial customer measure since it provides an external view of the system and has a direct effect on many of the other performance indicators. Dissatisfied users may opt not to use the system at all preferring to borrow books from elsewhere, buy their own copy of important books or sub-lend from other users. The loss of these users may well 
give a distorted view of system performance as measured by other means. For example, fewer library users may well improve response times within the library, reduce the number of reservations for books (indicating an adequate book supply) and reduce the number of issues of important core text books. Consequently, predicting the demand, or potential demand, for new titles and assessing the need for duplicate copies becomes difficult as unsatisfied demand is not measurable through book circulation statistics. They only measure what did happen and not what might have happened.

In this paper, we examine how systems thinking and, in particular, qualitative system dynamics modelling can be applied to an academic library system, with a view to exploring how commonly occurring system archetypes well known from the systems literature can also be found within models of user behaviour in academic library systems. Recognising that these archetypes exist can help to explain aspects of user behaviour and gain a better understanding of how changes to the library system in terms of changed parameter values (such as the loan period) or changed policy (perhaps in the allocation of scarce resources) might be reflected in changed user behaviour. A key component in the application of the systems dynamics modelling approach is the recognition of the feedback structures that very often in life control behaviour. Understanding the nature of feedback is often the key to understanding system behaviour and the modeller can gain insights about the system under study just by considering these qualitative models. Since this article is concerned with illustrating some of the basic concepts of system dynamics and illustrating archetypal structures in the context of library systems, a full system description or computer-based simulation model has not been described. However, such a model would be built if it was necessary to conduct a more detailed analysis of system performance and system response to change and this would follow from the qualitative modelling stage.

\section{System modelling in library management}

There exists considerable literature describing the quantitative models that have been developed and applied to library systems since the early work of the 1960s. The approaches adopted have been many and varied often depending on the particular part of the system being modelled, and a number of texts summarise the application of management science techniques to libraries (Rowley and Rowley, 1981; Kraft and Boyce, 1991). During the 1960s and 1970s there was a great deal of interest in applying the tools of management science to library systems and classic examples of studies from that period illustrate the range of models that were developed: these include deterministic, stochastic and simulation approaches to modelling. In this short review we shall consider only the last of these as simulation models are those that attempt a full system description and attempt to look at library systems from a holistic, systems perspective.

\section{As far back as 1968 Leimkuhler observed that}

.. where dynamic systems involve complex networks of many random and partially random components with a high degree of interdependence it may not be practical to attempt a completely analytic description, but better to simulate the system behaviour on a computer. (Leimkuhler, 1968) 
Simulation is one of the most frequently used decision support techniques and there have been many examples of simulation use since the 1970s. At this time computing technology became cheaper yet powerful enough for sophisticated desk-top applications. Early examples of these have included applications by Baker and Nance (1970), Arms and Walter (1974) and Thomas and Wight (1976). Baker and Nance tried to relate library activities to user needs in terms of lending books, providing service personnel and providing storage and study space. Circulation analysis was undertaken by Shaw (1976) but the most comprehensive early simulation work in this area has probably been that of Buckland (1975). Buckland's work related together the loan period, duplication policy, book popularity and satisfaction levels (as measured by the probability of finding a required book on the shelf). The work resulted in the implementation of a variable loan and duplication policy in the University of Lancaster library the outcome being an increase in the satisfaction level measured six months later. The increase was not, however, sustainable and we shall return to this later.

Much of the quantitative modelling work undertaken since this early pioneering work has used current or forecast book demand and circulation rates. Both of these necessarily imply no change in the behaviour of users even though changing library policy (e.g. changing the loan period or adding additional copies to the book stock) will have changed the system, user satisfaction within the system and therefore the way users will interact with the system. In this sense many of the models are essentially static. Furthermore, if we consider the simulation models that have been developed then these too have tended to avoid capturing the difficult behavioural elements of the system. Main alluded to the problem when she said of the Baker and Nance models:

Underlying these studies, and largely ignored, is a very real problem which arises when computer simulation is applied to library procedures. The mechanical, routine parts of work procedures can be described and simulated relatively easily. Human aspects, the social and political dimensions, pose a more difficult challenge, and have all to often been overlooked. (Main, 1987, 289)

In other words, quantitative and simulation models have tended to ignore human interaction and assume complete objectivity in decision making and this has caused some questioning of the confidence we should place in these early simulation models (Dahlin, 1991; Buchanan, Henig and Henig, 1998). A more complete picture would be provided by trying to model and therefore predict user behaviour within a system so that the real effect of system change can be investigated through the changed behaviour of humans within the system. Buckland (1975) also recognises this in his mention of the 'feedback loops' that can exist within library systems and this type of modelling can be readily approached through the application of system dynamics, a branch of modelling specifically oriented towards the study of feedback and that seems to have found little application in this area to date.

\section{System dynamics}

System Dynamics was developed in the late 1950s by Jay Forrester at the Sloan School of Management, MIT (Forrester, 1961). He contended that the (then 
developing) field of management science had a number of significant weaknesses that prevented it from being a powerful tool in management decision support. In particular, he felt that it was too mathematically oriented and focussed too much on optimisation and mathematical analysis to be of help in attacking broad, strategic management problems and that it could really only be applied to relatively simple problems taken in isolation rather than helping to understand the more complex interrelationships between corporate functions. Instead, Forrester preferred a closed-loop approach to the practice of management science in that taking decisions will automatically impact the corporate environment of the decision maker and changes to the environment will provide input to further decisions. This view is described by Coyle (1996) as the information/ action/ consequence paradigm in which "Information produces actions which have consequences, generating further information and actions and so on." Of course, this process does not happen in an instant and there may be significant delays between any of the three components, but the loop acting over time describes dynamic behaviour and these loops form the core of what is known as system dynamics.

The loop just described incorporates feedback and there are two types of feedback loop; the positive feedback loop and the negative feedback loop. A positive feedback loop (also known as a reinforcing loop) acts as a growth driver within a system. For example, suppose a university faculty has to attract and maintain a minimum number of students on its courses for financial security. If it can reduce the failure and withdrawal rate of students then it will increase the number of students progressing from one year to the next which means that fewer new students need to be recruited each year which produces more competition for places so that only the better students are selected which further reduces failure and withdrawal rates and so on. Thus the quality of the students is driven upwards as time progresses. Unfortunately, the same type of behaviour can also act in the opposite direction so that if failure and withdrawal rates start to increase, more new students need to be recruited so that the faculty can be less selective and this may further increase failure and withdrawal rates and so on. Positive feedback loops are sometimes colloquially known as 'virtuous' or 'vicious' circles depending on their action (Martin, 2008).

Negative feedback loops on the other hand are sometimes also called 'balancing' or 'goal seeking' loops. The effect of this negative feedback is to try and use the structure of the system to reduce the difference between the actual and some desired state of the system hence the 'balancing' or 'goal seeking' terminology. An oft-quoted example here is a central heating system in which the desired temperature of a room is set on a thermostat which also monitors the actual temperature and will then cause heat to be pumped into the room if the actual temperature is too low or allow the room to cool down if the actual temperature is too high. The effect of this action is constantly monitored to inform further action. Within the library context, we might purchase extra copies of a key text book which increases availability and encourages more users to use the library. After a while though the increased user numbers have utilised the extra copies, reservation lists grow and users are unable to find copies on the shelf so user numbers stabilise again and the system has re-balanced itself. It could be argued 
that the effects observed by Buckland (1975) on implementation of the variable loan and duplication policy at the University of Lancaster is a further example of a balancing loop in action. The impact of the new policy increases the perceived efficiency of the library system thus increasing user satisfaction but also encouraging further usage of the book stock which in time re-balances the system so that satisfaction levels eventually settle again.

It is sometimes the case that we are interested in examining and understanding some observed real system behaviour and this is termed 'reference mode behaviour'. Initial description of a system in terms of its loops can be undertaken using influence diagrams. An influence diagram is a qualitative, diagrammatic model that shows the structure of the system and its loops and helps us to understand the dynamic behaviour of a system and will hopefully give some guidance as to the causes of the reference mode behaviour. It would also form the basis for the building of quantitative models which might (although this is not always necessary) follow later. We now describe how influence diagrams representing system dynamics archetypes might be used to begin to describe and understand user behaviour within an academic library. As such we are undertaking qualitative modelling rather than quantitative modelling and this can yield useful insights into system behaviour.

\section{Influence diagrams and system archetypes}

Influence diagrams can be used to describe system archetypes and these are generic structures that have been observed by system modellers in a variety of business and management systems. These archetypes have been described in the context of systems modelling by Senge (1990) and have been used in a variety of educational settings where systems thinking is to be encouraged. Such an example is that of Atwater and Pittman (2006) who describe the usage of systemic thinking within business and management education. We will now discuss the structure of five system archetypes that occur within the context of library models but first we show the simplest structures of a reinforcing loop (positive feedback) and a balancing loop (negative feedback). These are shown in figure 1. 


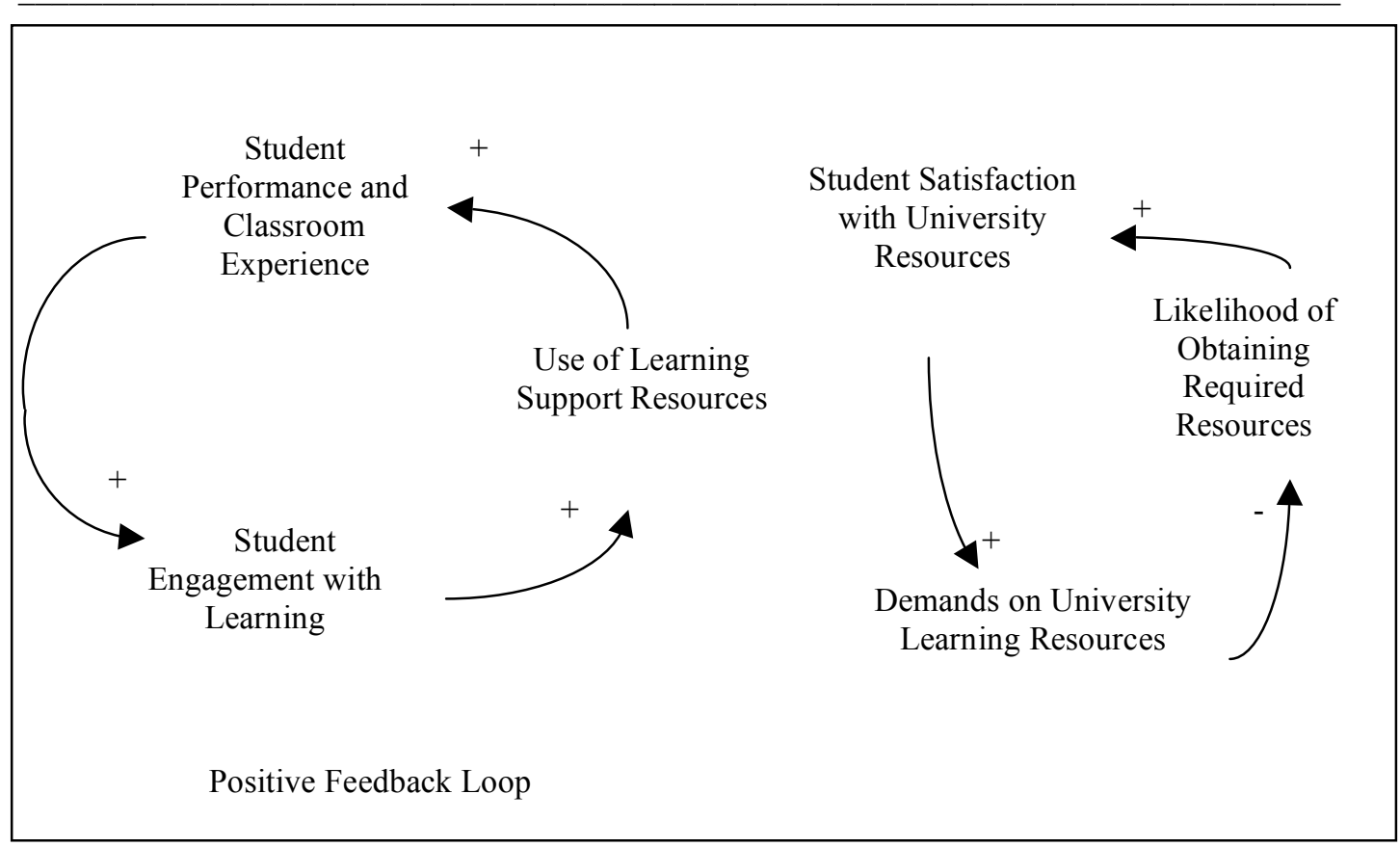

Figure 1: Positive and Negative feedback Loops

Arrows on the diagram illustrate linked concepts with the dynamic of the linkage shown using a ' + ' or '-' sign. The ' + ' sign indicates that increasing (decreasing) the first concept increases (decreases) the linked concept. The '-' sign indicates that increasing (decreasing) the first concept decreases (increases) the linked concept.

The first illustration is of the re-enforcing loop which is commonly describes as either a virtuous or vicious circle depending on whether the ever increasing (or decreasing) state benefits the system or not. In this loop there is no limiting process to restrain the state change. In our example based on recent research results (Warwick, 2009) all the links are positive indicating that increasing student engagement with learning will increase the demand for learning support materials (from all sources) and this will increase student performance and classroom experience which in turn encourages greater student engagement. Thus the loop is reinforcing. Note, however, that the loop could promote decreasing states in that if use of learning resources declines (perhaps due to non-availability) then performance will decline and this erodes student engagement which leads to a further decline in the use of learning resources.

The second illustration is of the balancing loop, in which the system is designed to move towards some (usually) pre-defined target or goal. In our example, increased use of university learning resources reduces the likelihood of obtaining the resources (due to greater competition) and this reduces satisfaction with the provision of resources and reduces further demands for the resources as students seek alternatives.

These two types of feedback loop occur commonly in system dynamic models as they describe the core feedback processes. When they are combined then we generate more complex structures and some of these are quite common in a variety of contexts and so have become well understood and termed archetypes. In a recent paper by Eric Wolstenholme (2004) the importance of identifying such 
archetypes is discussed and he comments that "Archetypes can assist model conceptualisation by virtue of their isomorphic properties to transfer thinking from one domain to another. Archetypes are also useful to communicate modelling insights by collapsing a model down to its basic loops." Furthermore, and in relation to the modelling process itself, he states that "In practice, it is often beneficial to use the archetypes in parallel throughout the process to guide highlevel thinking whilst detailed modelling is taking place." We now discuss the five examples of archetypal structures.

\section{Limits to Success}

This is the combination of a reinforcing loop and a balancing loop so that although the reinforcing loop may be increasing desired behaviour, the linked balancing loop restricts the growth often due to the limited nature of resources. This is shown in figure 2 .

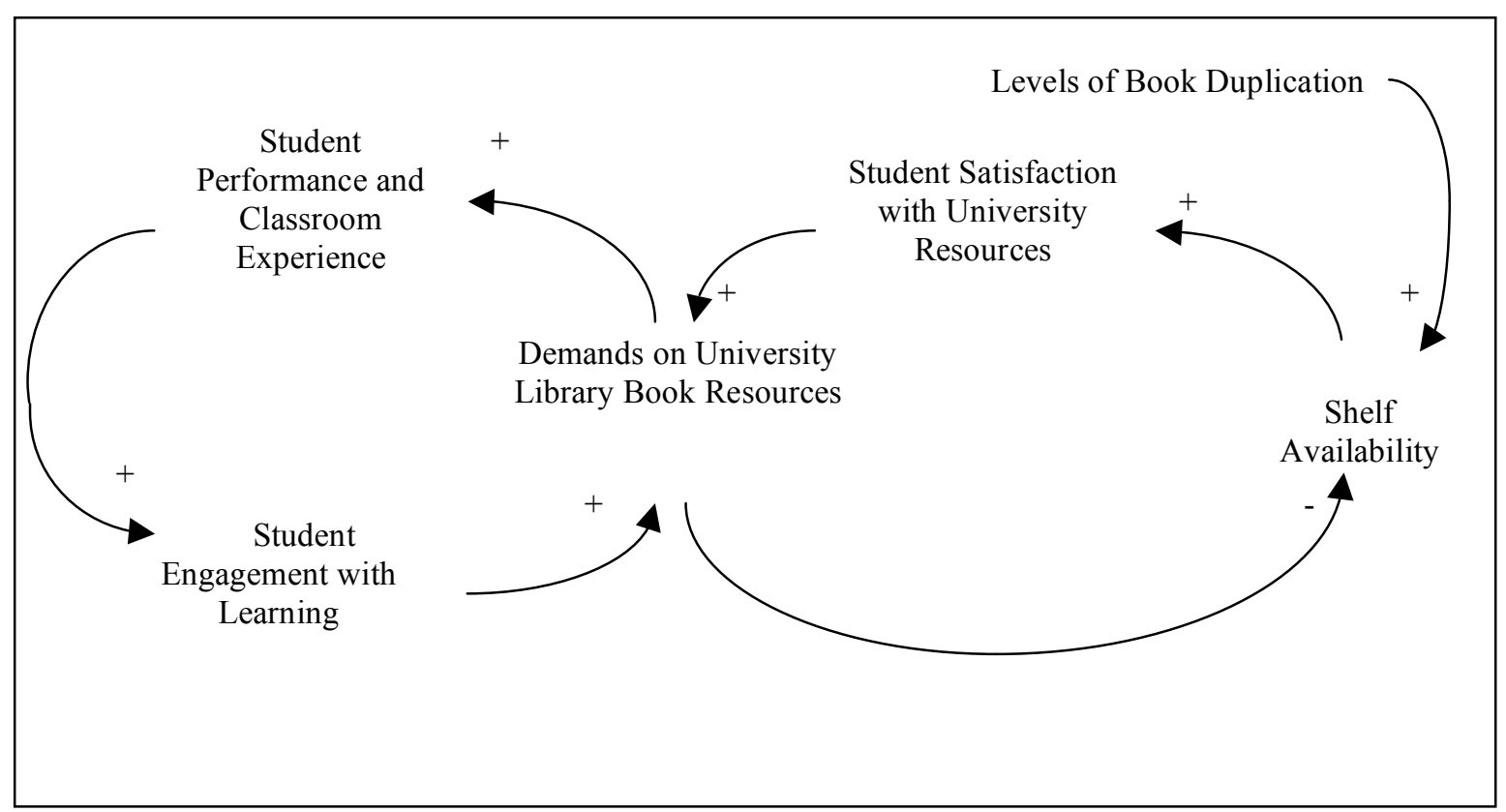

Figure 2: Limits to Success Archetype

In this example we can see that the growth in student engagement with learning leads to increased demand on university learning resources. However the limited university learning resources available restrict the growth in demand for university learning resources and so further growth in student engagement is not possible.

\section{Fixes that Fail}

This archetype represents situations under which a problem is identified and a solution (fix) implemented that reduces the problem. Unfortunately, the fix has unintended consequences that in fact acts to exacerbate the problem and nullify that implemented solution. This is exemplified by the illustration in figure 3 . 


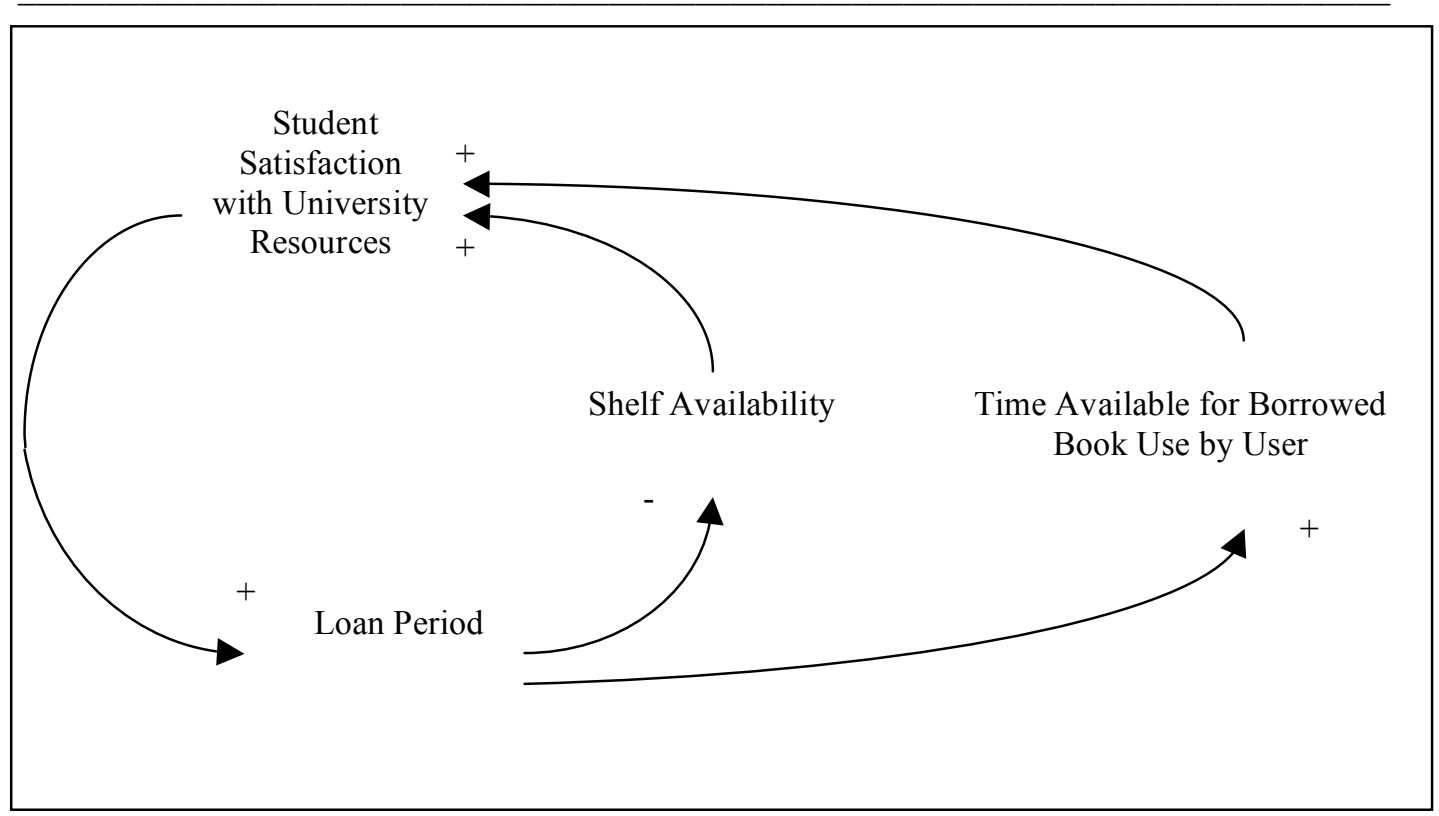

Figure 3: Fixes that Fail Archetype

In this example, low book shelf availability is producing low user satisfaction so to fix this the loan period for popular titles is reduced with intention of improving shelf availability and hence user satisfaction. Unfortunately, reducing the loan period means that users can get less use from a book before having to return it which then leads to frustration and reduced user satisfaction. This effect may be particularly acute for highly recommended core text books.

\section{Shifting the Burden}

This archetype represents a situation in which a problem has a fundamental solution which can alleviate the problem but which incurs a significant delay or effort. Other solutions can alleviate the symptoms of the problem (without attacking the root cause) and because symptoms are reduced, the urgency of finding and applying the fundamental solution is also reduced. Thus the burden is shifted and the symptomatic solution is preferred to the more long term fundamental solution. We illustrate this in figure 4 using a similar example to that in figure 3.

In figure 4, shortening the loan period is effective in increasing shelf satisfaction and increasing circulation rates which improves user satisfaction. In fact, the preferred way to improve student satisfaction is to bid for a greater proportion of university resources and use the money to provide more duplicate copies which increases shelf satisfaction and allows loan periods to be held constant. However, the inevitable delay in bidding processes for university funding (often undertaken on an annual basis) means that the apparent benefits of shortening the loan period as an alternative to improved duplication levels becomes an attractive option and may weaken the desire to seek more resources. 


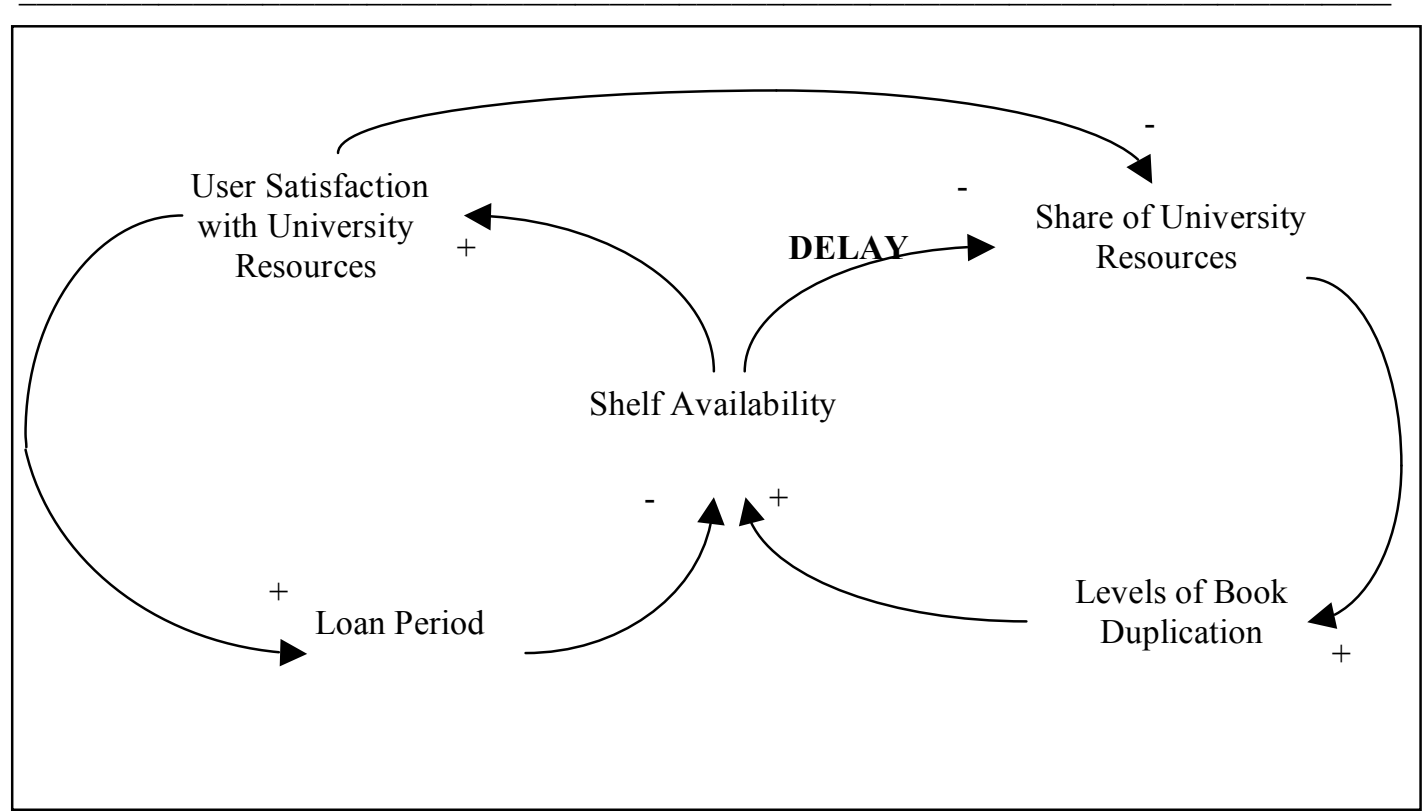

Figure 4: Shifting the Burden Archetype

\section{Drifting Goals}

This archetype allows us to deal with situations in which a person's expectations of system performance do not match their actual experience of system performance. In the case of a library, users will often attempt various actions within the library (making a reservation for example) with an idea in mind of the expected response of the library system to that action. If the chances of a successful transaction are small then the action is unlikely to be attempted so that, for example, if reservation waiting times are expected to be long, then it is unlikely that a reservation would be made.

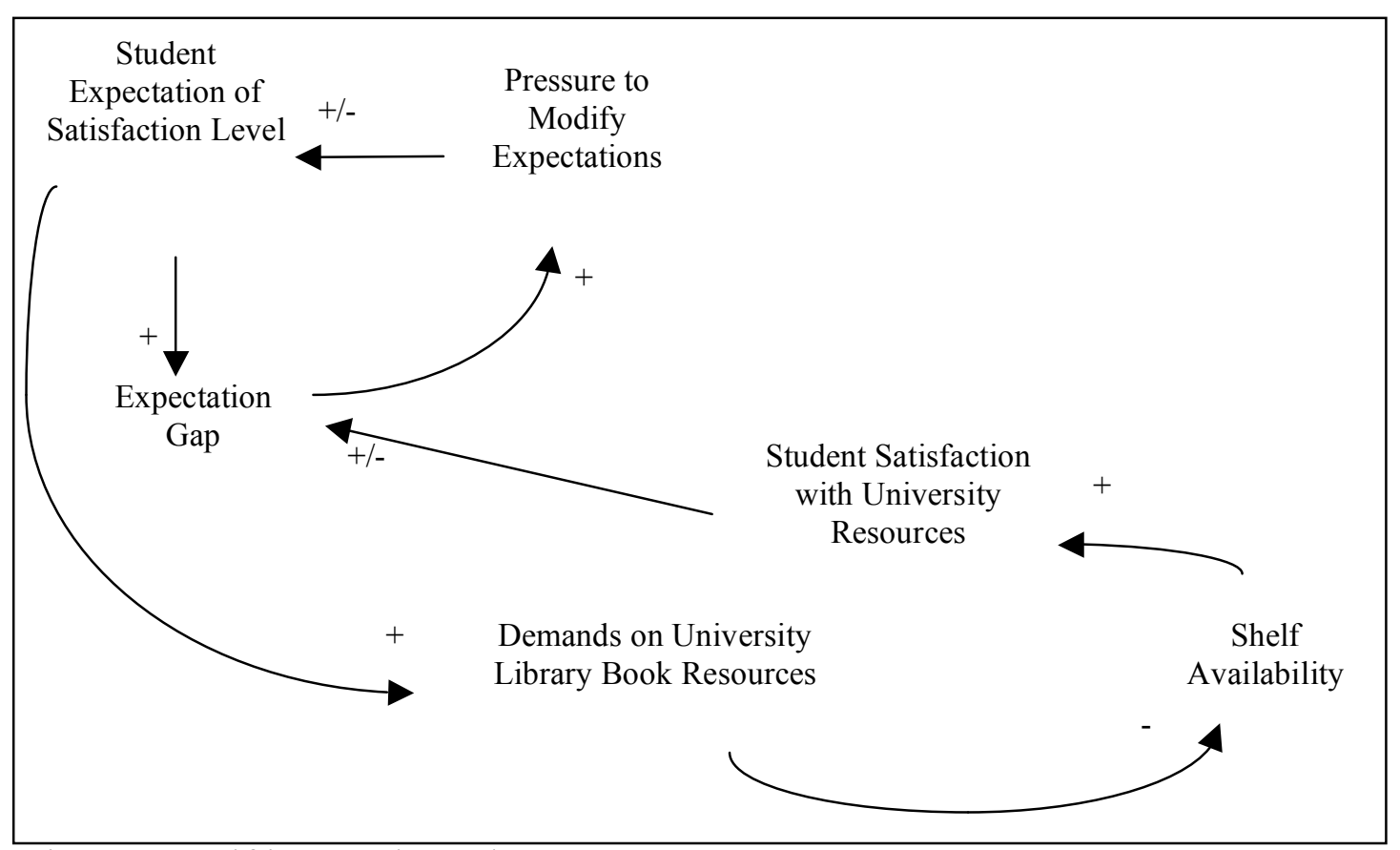

Figure 5: Drifting Goals Archetype 
Now, if expectations of system performance are not matched by actual performance in the eyes of the user then an expectations gap opens up and there are two possible consequences. Either the user will try again to get satisfaction from the system and hence close the gap, or the user's expectations of performance are modified. When expectations of library performance are low then it is likely that alternative sources of resources (other libraries, buying books, using the internet etc.) will be sought. In figure 5, two links are labelled with a $+/-$ indicating that the direction of action of the link could be in either direction. For example, if expectations of satisfaction are higher than actual satisfaction then raising actual satisfaction will reduce the gap. On the other hand, if expectations of satisfaction are lower than actual satisfaction, then raising actual expectations will increase the gap.

\section{Growth and Underinvestment}

This more complex archetype models the situation in which desired system growth is hindered by underinvestment which reduces system performance. In this case we have three intersecting feedback loops as shown in figure 6 .

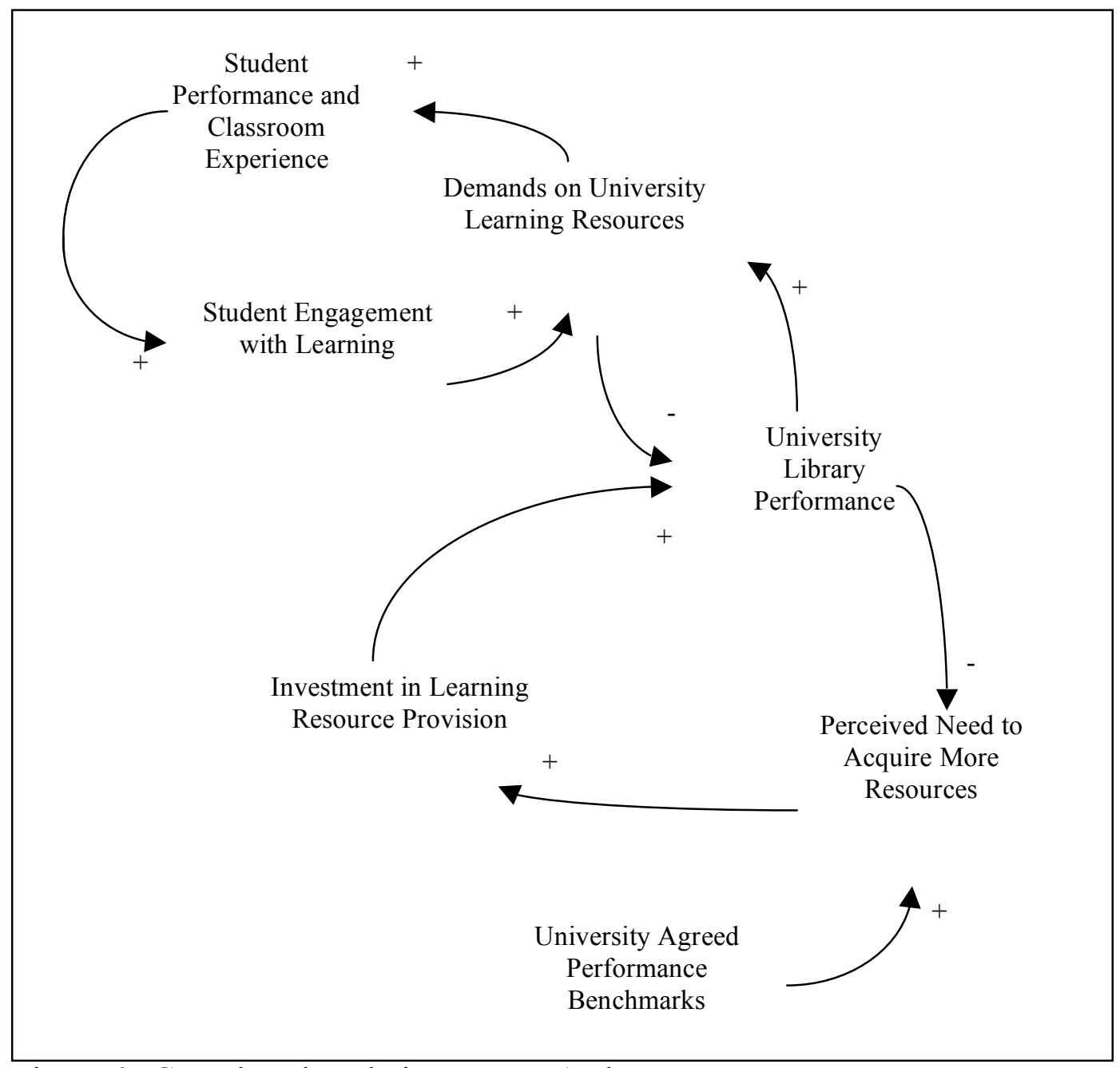

Figure 6: Growth and Underinvestment Archetype 
It is possible now to combine some of these archetypes together to form a model of part of the library system and an example of this is provided in figure 7.

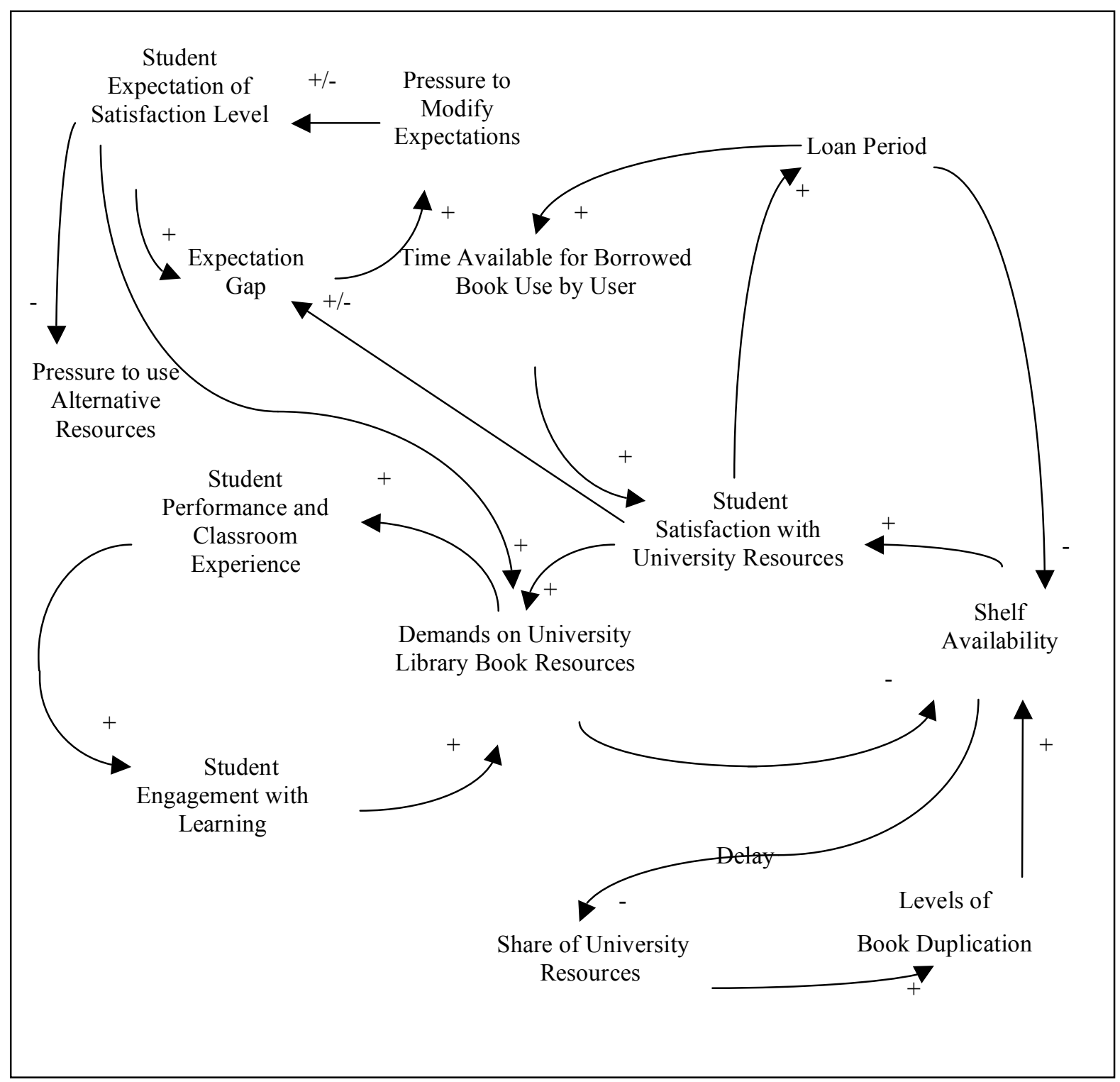

Figure 7: Combination of Archetypes

In figure 7 we have combined four of the archetypes described above around the core student learning feedback process. This now gives a more complete picture of the dynamics and interactions of the core cause and effect relationships at play and such a qualitative model can be used to draw some insights about the effect of policy changes in library management.

\section{Qualitative analysis}

Model development using systems dynamics has been described as following distinct phases (Sterman, 2000) and these may be summarised as: 
Phase 1: this involves general problem articulation, boundary setting and data collection that helps to identify the key feedback structures. Also important here is the identification of 'reference mode behaviour' which is to say observed system behaviour that we might use the model to explore and, hopefully, explain.

Phase 2: a qualitative model is constructed that describes the causal relationships that exist between the key elements of the system identified from Phase 1. In some situations the qualitative model of Phase 2 can be further developed into a computer based simulation model allowing the behaviour of a system to be simulated over a designated period of time. This is not always appropriate (if, for example, data is difficult to obtain) and is not always necessary in order to describe and learn about system behaviour.

Phase 3: this involves examining the model to see if we can identify any feedback loops or other standard system archetypes, and whether the model can give explanations of the identified reference mode behaviour.

Phase 4: we use the model to gain insights and draw conclusions as to how the system might be made to operate more effectively or efficiently.

Looking at the complete diagram of figure 7 a number of major feedback loops are now present and these are not just those that operate within each archetype. By having the archetypes connected together new feedback loops have been introduced so that analysis of the model will involve not just consideration of the archetypes but also of these new looping structures.

We can now begin to use models such as this to suggest explanations of observed behaviour. We consider three examples from figure 7 .

First, if actual levels of student satisfaction are low and below student expectations of satisfaction, then the expectations gap opens and acts to reduce expectations of satisfaction levels. This reduces further demands made on the university library and encourages students to seek alternative sources of resources. Ironically, this reduction in demand on university library resources increases shelf availability over time and this then increases student satisfaction for those still using the university library.

Second, if student satisfaction is increased to a level higher than expected by students (for example the introduction of a variable loan and duplication policy as in the Lancaster study) then this again opens the expectations gap which encourages students to modify expectations of satisfaction upwards which increases demands made on the library resources as more students are encouraged to make use of the library so that over time shelf availability reduces again as does student satisfaction. This increase in satisfaction followed by a later reduction was observed in the Lancaster study.

Third, if the system is 'shocked' by making a significant change then we can follow the effects through the system. Suppose that the library is given an injection of funding which allows additional copies to be purchased. This will increase shelf availability and hence student satisfaction and, as we have described, encourage more students into the system. As satisfaction goes up we could then possibly increase loan periods so that more use can be made of 
borrowed books and satisfaction increased further. Of course, lengthening loan periods will also reduce shelf availability which puts downward pressure on satisfaction so these effects have to be balanced and more detailed modelling would be required to obtain the correct balance.

The influence diagram would now go through a process of refinement and possible expansion as relevant stakeholders discuss the implications of these interactions, the assumptions being made and the logic it expresses. If analysis of the loops at this stage indicates behaviour that has not been observed or which is felt to be clearly inappropriate then amendments will need to be made. Modelling of this type is not a precise science and the models themselves should go through twin processes of enrichment and elaboration (Morris, 1967) during which they are tested against what is observed in the real system and unrealistic assumptions removed through the addition of further detail to the model.

\section{Subsequent analysis}

The influence diagram, while useful in its own right in conveying some important general ideas, may only be the first stage of a full system dynamics project. Once this model is agreed then it is sometimes desirable to continue to build a computer-based simulation model so that the interactions can be observed over time and the detailed dynamic behaviour becomes explicit. Specialist software exists for the support of system dynamics models and once the computer-based model has been constructed and tested it can then be used for detailed policy analysis. This might involve experimentation with changed values of some of the parameters within the model (perhaps so that the current system is 'tuned' to operate as effectively as possible) or experimentation with changes to the structure of the system and hence the model. For example one might be interested in assessing the effect of a variable loan policy which changes the loan period in response to the demand rate. This would necessitate structural change to the model but the ability to carry out such experimentation is one of the key advantages to modelling of this type.

\section{Conclusion}

In recent years there has been great interest in system dynamics and in particular its application within the business and management domain. As we pass 50 years of system dynamics, the value of this style of analysis is now being recognised. Although engineers have long understood the ideas behind process and systems control, it has taken a while for management scientists to appreciate the possibilities generated by moving the methods to their own domain of application. Library systems provide an excellent opportunity to demonstrate the importance of understanding the role that feedback loops play in explaining dynamic behaviour and it is to be hoped that further work can be done in applying these ideas to the problems of library systems analysis.

This article has tried to highlight some of the strengths of this type of approach, in particular the study of system archetypes, and these may be summarised as follows: 
1. System dynamics is a methodology that is oriented towards explanation of system behaviour through an analysis of the feedback structure of the system;

2. It is applicable to situations where more conventional management science models may have limited applicability or power and so is a useful addition to the modeller's toolbox;

3. It incorporates the use of influence diagrams which in themselves have significant use in exploring and understanding the structure and behaviour of a system and in communicating these ideas to others;

4. Influence diagrams may be further developed into a full simulation model if detailed analysis and experimentation is required although this is not always appropriate; and

5. By concentrating on structure and dynamic behaviour, system dynamics can be useful for strategic planning as well as for planning more detailed operational activities.

Of course there are drawbacks to any approach to modelling such as this. Influence diagrams can be difficult to construct until experience has been gained, and any simulation model can be time consuming to build if mathematical relationships and statistical data are to be included. However, any human activity system is going to present challenges when analysis is required and system dynamics provides a set of tools that hopefully will be more widely exploited for the purposes of understanding library systems and in particular the behaviour of library users.

\section{References}

Arms, W. Y. and Walter, T. P. (1974) A simulation model for purchasing duplicate copies in a library, Journal of Library Automation 7, 73-82.

Atwater, J. B. and Pittman, P. H. (2006) Facilitating systemic thinking in business classes, Decision Science Journal of Innovative Education 4 (2), 273-292.

Baker, N. R. and Nance, R. E. (1970) Organisational analyses and simulation studies of university libraries: a methodological overview, Information Storage and Retrieval 5 (4), 153-168.

Buchanan, J. T., Henig, E. J. and Henig, M. I. (1998) Objectivity and subjectivity in the decision making process, Annals of Operations Research 80, 333-345.

Buckland, M. K. (1975) Book availability and the library user. London: Pergamon Press.

Coyle, R. G. (1996) System dynamics modelling: a practical approach. London: Chapman \& Hall.

Dahlin, T. C. (1991) Operations research and organisational decision making in academic libraries, Collection Management 14 (3/4), 49-60.

Forrester, J. W. (1961) Industrial dynamics. Cambridge MA: MIT Press. 
Kraft, D. H. and Boyce, B. R. (1991) Operations research for libraries and information agencies, San Diego, California: Academic Press.

Leimkuhler, F. F. (1968) Mathematical models for library systems analysis, Drexel Library Quarterly 4, 185-196.

Main, L. (1987) Computer simulation and library management, Journal of Information Science 13, 285-296.

Martin, G. (2008) The phrase finder. URL: http://www.phrases.org.uk [accessed 16.08.08].

Morris, W. T. (1967) The art of modelling, Management Science 13, 707-717.

Rowley, J. E. and Rowley, P. J. (1981) Operations Research. A tool for library management. Chicago: American Library Association.

Senge, P. M. (1990) The fifth discipline: the art and practice of the learning organisation. New York: Doubleday.

Shaw, W. M. (1976) Library user interface: a simulation of the circulation subsystem, Information Processing and Management, 12 (1), 77-91.

Sterman, D. J. (2000) Business dynamics: systems thinking and modelling for a complex world. Boston: McGraw-Hill Irwin.

Sterman, J. (2007) Exploring the next great frontier: system dynamics at 50, System Dynamics Review, 23 (2-3), 89-93.

Thomas, P. A. and Wight, T. (1976) Computer simulation and library management, EURIM II, A European Conference on the Application of Research in Information Services and Libraries. London: Aslib.

Warwick, J. (2009) A developing qualitative model diagnosing the learning behaviour of undergraduate computing students, to appear in Problems, Resources, Issues in Mathematics Undergraduate Studies.

Wolstenholme, E. (2004) Using generic system archetypes to support thinking and modelling, System Dynamics Review 20 (4), 341-356. 